\title{
Tangence
}

\section{Liminaire. Images de l'Amérindien au Canada francophone : littérature et image}

\section{Hélène Destrempes et Hans-Jürgen Lüsebrink}

Numéro 85, automne 2007

Images de l’Amérindien au Canada francophone : littérature et image

URI : https://id.erudit.org/iderudit/018605ar

DOI : https://doi.org/10.7202/018605ar

Aller au sommaire du numéro

Éditeur(s)

Tangence

ISSN

1189-4563 (imprimé)

1710-0305 (numérique)

Découvrir la revue

Citer ce document

Destrempes, H. \& Lüsebrink, H.-J. (2007). Liminaire. Images de l'Amérindien au Canada francophone : littérature et image. Tangence, (85), 5-11.

https://doi.org/10.7202/018605ar d'utilisation que vous pouvez consulter en ligne. 


\section{Liminaire}

\section{Images de l'Amérindien au Canada francophone: littérature et image}

Hélène Destrempes,

Université de Moncton

Hans-Jürgen Lüsebrink,

Université de Saarbrücken (Allemagne)

«L'Indien a toujours occupé une grande place dans la symbolique de la collectivité francophone ${ }^{1}{ }^{\prime}$, écrit Fernand Dumont dans sa Genèse de la société québécoise. Et il est vrai que cette figure, sous des formes les plus diverses et parfois même les plus inattendues, a occupé et occupe encore aujourd'hui une part significative des discours culturels et littéraires tant québécois que canadiens et européens.

Au-delà de leurs usages commerciaux, littéraires, artistiques ou philosophiques, ces diverses représentations de la figure de l'Amérindien ont en commun de questionner, non pas la référence d'origine (l'autochtone «en chair et en os»), mais plutôt le rapport que nous entretenons avec ces représentations mêmes et l'ensemble des intertextes qui soutiennent et alimentent cette image depuis la découverte des Amériques. Elles soulèvent aussi la question de la "préfiguration inventive», pour reprendre l'expression de Fernando Ainsa, c'est-à-dire de l'adéquation forcée entre ce qui est réalité et ce qu'on voudrait qu'elle soit. «Si la réalité est différente, affirme-t-il, les données recueillies sont réinterprétées et adaptées à l'espace souhaité au préalable [...] L'Amérique devait être ce qu'on en attendait ${ }^{2}$.»

La figure de l'Amérindien nous renvoie également à la problématique du Soi et de l'Autre, d'un «soi-idem» ou bien «ipse», pour reprendre la terminologie de Ricœur, d'un nous inclusif ou exclusif,

1. Fernand Dumont, Genèse de la société québécoise, Montréal, Boréal, 1993, p. 68.

2. Fernando Ainsa, «L’invention de l'Amérique», Diogène, Paris, nº 145, 1989, p. 107. 
d'une identité souvent en quête d'origine amérindienne, susceptible néanmoins de dérober ou d'absorber l'indianité à l'instar de la mante religieuse qui dévore ses proies. Les diverses manifestations et usages de la figure de l'Amérindien, ainsi que les débats qui les entourent, revêtent donc une importance particulière dans le cadre de l'affirmation des nations autochtones dans les Amériques et de leurs revendications territoriales, politiques et culturelles.

Ainsi mise en perspective, la perception des nations autochtones a subi, au cours des vingt dernières années, dans la littérature comme dans les médias, un double déplacement que les contributions publiées dans le présent dossier tentent de prendre en considération. Elle s'est, d'une part, indéniablement politisée, dans le sillage, entre autres, du vaste mouvement de contestation contre les projets de barrages hydro-électriques de la Baie James dans les années 1970 et 1980, du déroulement de la crise d'Oka en 1990, ainsi que des nombreuses revendications politiques et territoriales, que ce soit auprès des gouvernements ou devant les tribunaux, depuis les trente dernières années. La perception des nations autochtones s'est trouvée modifiée, d'autre part, par l'émergence d'une prise de parole proprement amérindienne dans des lieux, des médias et des formes de discours les plus variés; que l'on songe, par exemple, aux interventions sur la scène internationale, devant les Nations Unies et à l'Unesco, ou à la publication de journaux et de revues autochtones, au développement de sites Internet, ainsi qu'à la création d'un réseau de télévision proprement autochtone au Canada (APTN). Il convient de mentionner également les communiqués politiques des divers leaders et intervenants autochtones dans la presse et les médias canadiens et québécois, de même que la publication d'œuvres littéraires et historiographiques amérindiennes ${ }^{3}$ et le développement de manifestions culturelles s'adressant tant à un public amérindien que non amérindien, plus particulièrement dans les domaines du théâtre, de la chanson et du cinéma. De récents témoignages filmiques, tel le film Ullimi (qui signifie «Aujourd'hui» en inuktitut), présenté à Télé-Québec en mars $2007^{4}$, confirment le

3. Mentionnons, à cet effet, l'Histoire du Haut et du Bas-Canada de Bernard Assiniwi (Montréal, Leméac, coll. Ni-T'Chawama, Mon ami mon frère, 19731974, 3 v.); ainsi que les travaux de George E. Sioui, et notamment Pour une autohistoire amérindienne, Québec, Presses de l’Université Laval, 1990.

4. Ullimi, par Les Films de l'Isle, diffusé le 4 mars 2007 par Télé-Québec dans le cadre de l'émission «Questions de société». 
développement de ces nouveaux espaces discursif, littéraire et médiatique amérindiens où chaque manifestation, chaque œuvre, chaque témoignage contribue à mettre en valeur l'autoreprésentation des réalités amérindiennes longtemps oblitérées par le discours et les représentations de l'Autre.

La persistance de diverses représentations amérindiennes, détachées en partie ou en totalité de l'univers du référent, nous rappelle cependant la pertinence de se pencher, encore aujourd'hui, sur le rôle et la fonction de cette figure dans l'évolution de l'identité et de l'imaginaire culturel et politique québécois. Les travaux de Fernand Dumont, notamment sa Genèse de la société québécoise ${ }^{5}$ et de Gérard Bouchard, principalement sa Genèse des nations et des cultures du Nouveau Monde ${ }^{6}$, demeurent jusqu'à présent de précieuses sources de renseignements sur l'émergence de la figure de l'Indien au Canada français; il en va de même pour les études publiées sur la figure de l'Amérindien depuis le début des années 1970 jusqu'au milieu des années $1990^{7}$. Il convient également de citer, en ce domaine, les cinq tomes parus de La vie littéraire au Québec ${ }^{8}$, qui sont riches de nombreuses données sur la figure de l'Indien et restituent les textes littéraires dans le contexte tant de leur production que de leur réception.

Hormis Recherches amérindiennes du Québec, qui a consacré deux dossiers aux "Portraits d'Indiens» et à "L'Indien

5. Fernand Dumont, Genèse de la société québécoise, Montréal, Boréal, 1996.

6. Gérard Bouchard, Genèse des nations et des cultures du Nouveau Monde, Montréal, Boréal, 2000.

7. Nous songeons ici aux travaux de Donald B. Smith, Le «Sauvage» pendant la période de la Nouvelle-France, Montréal, Cahiers du Québec/Hurtubise HMH, 1974; de Sylvie Vincent et Bernard Arcand, L'image de l'Amérindien dans les manuels scolaires du Québec, Montréal, HMH, 1979; de l'équipe de recherche de Gilles Thérien, dont les travaux, dans une perspective sémiotique, ont donné lieu à la publication de l'ouvrage collectif intitulé Figures de l'Indien, Montréal, l'Hexagone, 1995; ainsi qu'aux textes regroupés dans Antonio Gomez-Moriana et Danièle Trottier (sous la dir. de), L'Indien, instance discursive, Candiac, Balzac, 1993; sans oublier les importants travaux de Maurice Lemire, grand spécialiste du XIX ${ }^{e}$ siècle québécois, et notamment son ouvrage intitulé Formation de l'imaginaire littéraire au Québec (1764-1867) (Montréal, l'Hexagone, 1993) dans lequel tout un chapitre est consacré à ce que l'auteur nomme le "mythos indien»; et de Louise Côté, Louis Tardivel et Denis Vaugeois, L'Indien généreux. Ce que le monde doit aux Amériques, Montréal, Boréal, 1992.

8. Maurice Lemire (sous la dir. de), La vie littéraire au Québec, Québec, Presses de l’Université Laval, 1991-2005. 
imaginaire ${ }^{9}$ ", aucune revue scientifique au Québec ne s'est néanmoins penchée sur cette problématique à la même période. On trouve bien, à l'occasion, des articles sur la littérature de la Nouvelle-France, sur celle du XIX ${ }^{e}$ siècle ou encore sur le métissage, où la figure de l'Indien est parfois évoquée, mais à notre connaissance aucun dossier n'a encore été consacré à l'étude de la figure de l'Amérindien dans la littérature et les médias au Québec ou au Canada français ${ }^{10}$.

Le présent dossier vise donc à combler cette lacune en offrant un ensemble de contributions où il est justement question de ces déplacements discursifs dont nous avons parlé plus haut, des divers enjeux qui y sont rattachés et ce, dans le cadre d'une histoire pluriséculaire de représentations occidentales des Amérindiens, de leur univers social et de leur culture.

La prise de parole contemporaine des Amérindiens, où la littérature et l'historiographie, mais aussi les arts visuels, le film et l'Internet jouent un rôle important, ne peut être détachée des formes de représentation antérieures non autochtones par rapport auxquelles elle se situe et prend position. Loin d'être homogènes, celles-ci se révèlent, comme les différentes études publiées ci-après le montrent, d'une grande variété, que déterminent autant des facteurs historiques et idéologiques que les structures discursives et médiatiques dans lesquelles elles s'inscrivent. Afin de tenir compte de cette hétérogénéité fondamentale, l'éventail des discours et médias analysés a été très largement découpé: outre les textes littéraires, les écrits coloniaux et le domaine de la peinture, nous avons également interrogé des corpus jusqu'ici peu explorés, voire totalement ignorés dans la recherche sur les représentations des Amérindiens, et notamment les almanachs populaires canadiensfrançais.

Le dossier commence par une étude de Réal Ouellet, qui porte plus particulièrement sur les chroniqueurs du XVII siècle. Dans cet article, l'auteur se penche sur la perception du monde amérindien à l'époque de la conquête et de la colonisation des Amériques, par

9. Recherches amérindiennes au Québec, Montréal, vol. XVII, nº 3 (L'Indien imaginaire, sous la dir. de Gilles Thérien), 1987; et Recherches amérindiennes au Québec, vol. XI, nº 4 (Portraits d'Indiens, sous la dir. d'Anne-Marie Sioui), 1981.

10. Le dossier de la revue Globe, vol. 8, n 1, 2005, sur «Les modernités amérindiennes et inuite», ne comporte pas non plus d'articles sur la représentation des autochtones dans la littérature et la culture québécoises. 
le biais de paradigmes anthropologiques comparatifs, confrontant et mettant en parallèle les premières nations de la Nouvelle-France et celles des Caraïbes. Ces paradigmes, qui évoquent des traditions de représentations mythologiques fort différentes, renvoient en effet à l'imaginaire de la société naturelle et à la figure du Sauvage philosophe (telles qu'on les trouve, par exemple, dans les œuvres de Lahontan), ainsi qu'à l'imaginaire biblique du Paradis terrestre évoqué dès le premier voyage de Christophe Colomb.

En s'intéressant, pour sa part, aux représentations de la figure de l'Amérindien dans la littérature canadienne-française du $\mathrm{XIX}^{\mathrm{e}}$ siècle, Hélène Destrempes s'interroge non pas sur les figures en soi, mais plutôt sur les contraintes architextuelles et narratives régissant l'élaboration et la mise en texte de ces figures. En s'inspirant des travaux du sociologue Jean-Jacques Simard sur les politiques gouvernementales dans le domaine des affaires autochtones, elle développe un nouveau modèle interprétatif fondé sur le concept de «réduction». Elle cherche d'abord à esquisser le potentiel heuristique de cette notion, avant de proposer une analyse de deux contraintes paratextuelles, soit les titres et les intertitres des œuvres mettant en scène des personnages amérindiens, qui ont conditionné, à leur façon, la mise en discours de la figure de l'Indien dans la littérature canadienne-française au XIX siècle.

En s'attachant à une période qui va de 1850 à 1950, la contribution de Hans-Jürgen Lüsebrink est centrée sur la représentation de l'Amérindien dans le corpus des almanachs canadiens-français. Son étude montre, entre autres, que la figure de l'Amérindien demeure, jusqu'au début du $\mathrm{xx}^{\mathrm{e}}$ siècle, très marginale et qu'elle n'est évoquée que de manière très stéréotypée. Elle devient néanmoins, dans les récits ethnographiques et les contes populaires publiés dans les almanachs, comme ceux du frère Marie-Victorin, par exemple, mais aussi de Corinne Rocheleau et de Rodolphe Girard, une composante majeure de l'identité canadiennefrançaise et de sa mémoire historique. L'auteur, par ailleurs, rappelle à juste titre que les almanachs ont formé un support médiatique dont l'importance socioculturelle a été de tout premier plan dans la diffusion de cette mémoire historique, atteignant un large public aussi bien dans les villes que dans les campagnes québécoises.

L'étude de Louise Vigneault porte plus particulièrement sur la résurgence du sujet autochtone dans la peinture canadiennefrançaise de la première moitié du XIX ${ }^{e}$ siècle et des milieux de 
l'avant-garde des années 1940. L'auteur aborde ainsi, en un premier temps, l'œuvre d'Antoine Plamondon. Elle analyse, en outre, comment ce peintre s'est inspiré des canons romantiques de représentation de l'Amérindien et de quelle façon il a incité l'Amérindien qu'il avait portraituré, Zacharie Vincent, à devenir luimême peintre. Il s'agit d'un exemple fascinant qui témoigne de l'établissement de paradigmes nouveaux au sein du discours culturel canadien-français, où la figure de l'autochtone commence à incarner de multiples formes de rupture et de résistance. Dans la seconde partie de cet article, Louise Vigneault s'intéresse plus particulièrement aux rapports entre l'avant-garde artistique et les références à une indianité de résistance, chez les automatistes par exemple, qui se sont reconnus dans les luttes et le désir d'autonomie des populations autochtones.

Dans un article portant sur la figure de l'Amérindien et l'évolution de l'américanité dans le roman québécois depuis 1945, Jean Morency explique, pour sa part, comment l'image de l'Amérindien, bien qu'elle soit loin d'être omniprésente dans le roman québécois au $\mathrm{XX}^{\mathrm{e}}$ siècle, a néanmoins joué un rôle significatif dans l'évolution de certaines thématiques romanesques, comme celles des grands espaces et de la nature rédemptrice. Les romanciers québécois ont, de fait, souvent attribué à la figure de l'Amérindien une fonction symbolique dans le mouvement de prise de conscience de leur appartenance au continent américain, qui s'est ainsi révélée indissociable de l'expression et de la revendication d'une identité nord-américaine. L'article vise à dégager quelques-uns des avatars de l'image de l'Amérindien dans le roman québécois de 1945 à nos jours, avatars qui ont été regroupés en deux grands ensembles: d'un côté les Indiens proprement dits, et d'un autre côté les Métis et les «faux Indiens».

Emmanuelle Tremblay propose, enfin, un article sur les significations symboliques de la figure de l'Indien, telle que celle-ci apparaît dans Les enfantômes (1976) de Réjean Ducharme. Elle y montre, entre autres, que la fonction narrative de cette figure revêt une dimension critique qui se donne à lire tout particulièrement dans les représentations romanesques de la marginalité que la modernité littéraire reprend à son compte sur le plan de l'écriture en fonction d'une rhétorique que Ducharme désigne sous le nom d' «huronie». L'analyse vise à rendre manifeste comment cette dernière équivaut à une appropriation de l'indianité en raison de la liberté langagière qu'elle autorise, de la déconstruction du lieu 
commun dont elle procède, ainsi que de l'hybridité et de l'altérité qu'elle génère. Elle permet également de spécifier en quoi l'huronisation relève d'un principe de médiation entre les référents culturels convoqués (sérieux/populaires; français/québécois/ anglo-saxons) en raison du passage de l'un à l'autre, fondant ainsi le caractère ambigu de l'appartenance identitaire dans le texte ducharmien.

Finalement, sans naturellement prétendre épuiser tous les enjeux rattachés à la représentation de la figure de l'Amérindien dans la littérature et les médias au Canada français, l'ensemble de contributions offre néanmoins un éventail d'études qui permet à la fois de reconsidérer l'importance de cette figure au sein de l'imaginaire littéraire et culturel québécois, et de mieux évaluer la pertinence d'approfondir la recherche en ce domaine au Québec comme au Canada. 